\title{
How Would we Recognize a Martensitic Transformation if it Bumped into us on a Dark \& Austy Night?
}

\author{
P.C. Clapp
}

Center for Materials Simulation, Institute of Materials Science, University of Connecticut, 97 N. Eagleville Road, Storrs, CT 06269-3136, U.S.A.

\begin{abstract}
The history of the meaning of "martensitic transformation" over the past forty years is briefly reviewed, and an area of common agreement is identified centered on the concepts of "cooperative atomic motion" and "shape change". A search is made for the minimum elements required in a definition that will be simple, compact, precise and easily tested. It is argued that cooperative atomic motion implies acoustic emission, and that this could provide an important operational element in the definition. A distillation of past definitions, with this new ingredient added yields, as a proposed irreducible definition: A martensitic transformation involves a cooperative motion of a set of atoms across an interface causing a shape change and sound.
\end{abstract}

\section{Introduction}

It may well be asked why it is important to find a definition of "martensitic transformation" any more precise than the myriad of examples already present in the literature. The usefulness of a clear definition has at least four components:

a) It helps us think more accurately about the subject

b) It facilitates discussion by ensuring that everyone is on the same "wavelength"

c) It makes teaching of the subject easier and improves student retention

d) It provides a useful indicator of related knowledge and phenomena

History has also shown that the scientific definitions that have been the most useful over extended periods of time are those that are:

1) Simple, compact and precise

2) Easily tested

Most phase transformations that we are familiar with are indeed compactly defined (i.e. a single sentence usually suffices) and can be detected unambiguously with one (or at most two) straightforward experimental measurements. The following list of examples is offered as an exercise for the reader: Order-disorder, Ferromagnetic, Ferroelectric, Superconducting, Melting, Freezing, Clustering (precipitation), Superfluid.

However, more than a century after "martensite" in steel was first observed ${ }^{1}$, and exactly one hundred years after martensite was named by M. F. Osmond 2 (in honor of A. Martens), with the subsequent generalization of the term "martensitic transformation" to similar phenomena in other alloys $^{3}$ (not without many objections at the time), it is still difficult to explain to a neophyte (or even an expert!) what exactly a martensitic transformation is, and to give an operational definition that can be easily translated to the laboratory. In fact, discussions of what a proper definition should be often resemble the fabled debate of the seven blind men encountering an elephant! Why is this, and what can be done about it?

Before tackling this thorny question it is worth taking some satisfaction in the considerable success that the crystallographic theory ${ }^{4,5}$ (and apparently the linear ${ }^{6}$ and non-linear ${ }^{7}$ thermoelastic continuum extensions of it) have had in our ability to analyze and predict a wide variety of crystallographic relations and morphological features of martensitic systems. This whole body of analysis may be referred to collectively as continuum crystallographic theories. This approach, which ignores any details of atomic movements, interface structure or transformation mechanisms has been, and continues to be, a remarkable achievement.

Certainly one of the stumbling blocks in defining a martensitic transformation is that there is no obvious "order parameter" associated with it, such as one has with ferromagnetic transformations (magnetic moment), order-disorder (long range order parameter), etc. Of course, one always has the possibility of using the volume fraction transformed as such an order parameter, analogous to the use of the fractional density of superconducting electrons for superconducting transformations, but this is a much more erratic quantity (markedly dependent on sample history) in the case of interest here. 


\section{Review of Previous Definitions}

Many definitions of a "martensitic transformation" have been offered in the literature, and it is useful to review them to see to what extent one can find a common ground. To quote just some of the more familiar in more or less chronological order, with what I perceive to be the key words or phrases underlined:

Bilby \& Christian 8 - "A structural transformation is classed as martensitic if the atoms on a primitive lattice defined by a selected unit cell of the parent structure move to positions on a primitive lattice defined by some unit cell of the product structure, in such a way that the displacements constitute a homogeneous deformation. This deformation may be different in adjacent small regions."

Kaufman and Cohen ${ }^{9}$ - Martensitic reactions are displacive or shear-like. They entail a cooperative movement of atoms such that the region in question undergoes a transformation strain or change in shape. Fiducial lines on a pre-polished surface are sheared over, and the free surface of the region is tilted out of the plane of polish. No diffusion or interchange of atoms is involved in this process. The transformation product inherits the composition of the parent phase, and may be atomically ordered if the latter contains a superlattice.

Christian ${ }^{10}$ - The martensitic reaction thus occurs because of the existence of an easy growth mechanism, not requiring atomic diffusion, which leads to the rapid production of a new phase and a net lowering of free energy. This question of growth mechanism is the central feature of a martensitic change, and illustrates the importance of studies of the nature of the interface in such a reaction (see also Fig. 1).

Lieberman"1 - "The generally accepted geometric or crystallographic features that characterize martensitic transformations include:

(i) A shape deformation which results in surface upheavals in a polished reference surface of the parent or a sharp dihedral angle between parent and product indicating that, macroscopically, the transformation consists of a shear on an undistorted plane and a possible volume change perpendicular to it, i.e., an invariant plane strain.

(ii) The transformation is heterogeneous since it is accompanied by the formation of new interfaces.

(iii) The interface or habit plane separating parent and product in a partially transformed crystal is on the average undistorted.

(iv) The habit plane is generally irrational with its normal having direction cosines characteristic of the material.

(v) A lattice correspondence exists between a unit cell in the parent and one in the product, which is not necessarily unique.

(vi) There is a rather precise orientation relationship between principal directions and planes in the two phases on either side of the interface.

(vii) There is a discontinuous change in some parameter, such as the lattice constants, during the transformation.

(viii) Atoms frequently move less than an interatomic distance and hence nearest neighbours (or at least atomic sites) are maintained as far as possible during the change in crystal structure.

(ix) There is usually a change in crystal symmetry and most of the time no change in composition.

(x) There is frequently evidence of a fine inhomogeneous structure such as slip or twinning, in the parent or product phase (usually the latter) which does not alter the lattice, i.e. it is a lattice-invariant shear.

(xi) Generally there is a temperature hysteresis and the transformation is polymorphic. Sometimes a series of transformations of decreasing symmetry is observed as the temperature is decreased, such as cubic to tetragonal to orthorhombic in some of the actinides and in some perovskite-type crystals." 
and also, "The classification system herein proposed differentiates according to the relaxation of item (viii) on the movement of the atoms of at least one of the species. If no atoms move more than an interatomic distance, the transformation is here designated as orthomartensitic. If some atoms can move an interatomic distance or so (very-short-range diffusion) while atomic sites are maintained and composition remains invariant (as in ordering), then the transformation is called paramartensitic. If at least one of the species moves over large distances and long-range diffusion occurs to produce a composition change, the transformation is termed quasi-martensitic. In all these cases the crystallographic features characteristic of a martensitic transformation are exhibited, however."

Clark and Wayman"12 - "A martensitic transformation is in general described by an invariant-plane strain relief effect at a free surface, an irrational habit plane and orientation relationship, a lattice correspondence between substitutional atoms which implies the absence of long-range diffusion, and the presence of an internal inhomogeneity such as twinning, all of these features being uniquely related in accordance with the phenomenological crystallographic theory, according to which this body of information is completely internally consistent.

A working definition such as this is precise, encompassing, and excludes those occasional transformations with some, but not all, matching characteristics. At the same time, however, we should point out that such a description by its (phenomenological) nature really gives no information on mechanistic details, other than the implied coordinated or "military" atomic movements and the likelihood of a semi-coherent dislocation interface."

Nishiyama ${ }^{13}$ - "A martensitic transformation is a phase transformation that occurs by cooperative atomic movements. The product of a martensitic transformation is martensite. That a given structure is produced by a martensitic transformation can be confirmed by the existence of the various characteristics that have been discussed, especially the diffusionless character, the surface relief, and the presence of many lattice imperfections. Such characteristics are therefore criteria for the existence of martensite."

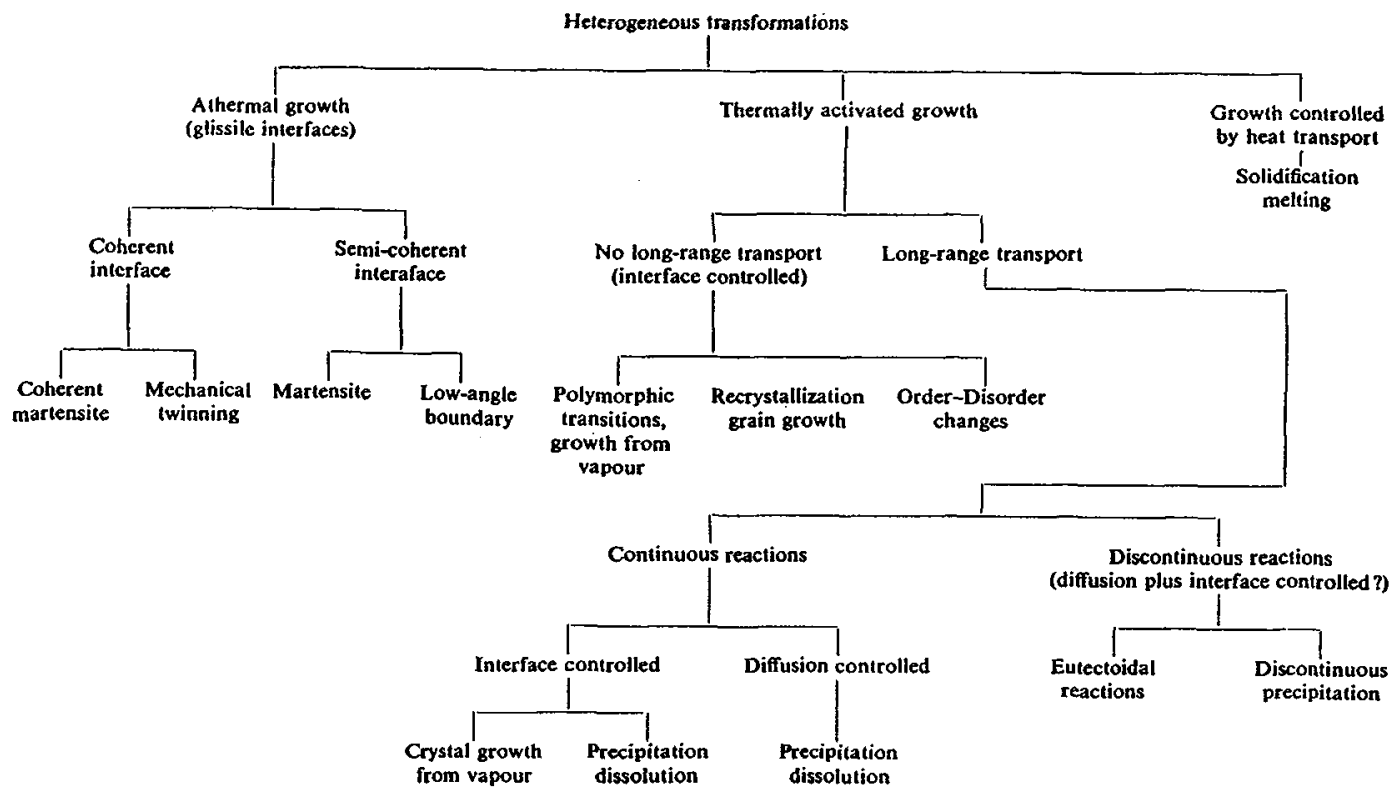

Fig. 1 - The classification of phase transformations proposed by Christian10 
Cohen, Olson and Clapp 14 - "A martensitic transformation is a lattice-distortive, virtually diffusionless structural change having a dominant deviatoric component and associated shape change such that strain energy dominates the kinetics and morphology during the transformation." (see also Fig. 2).

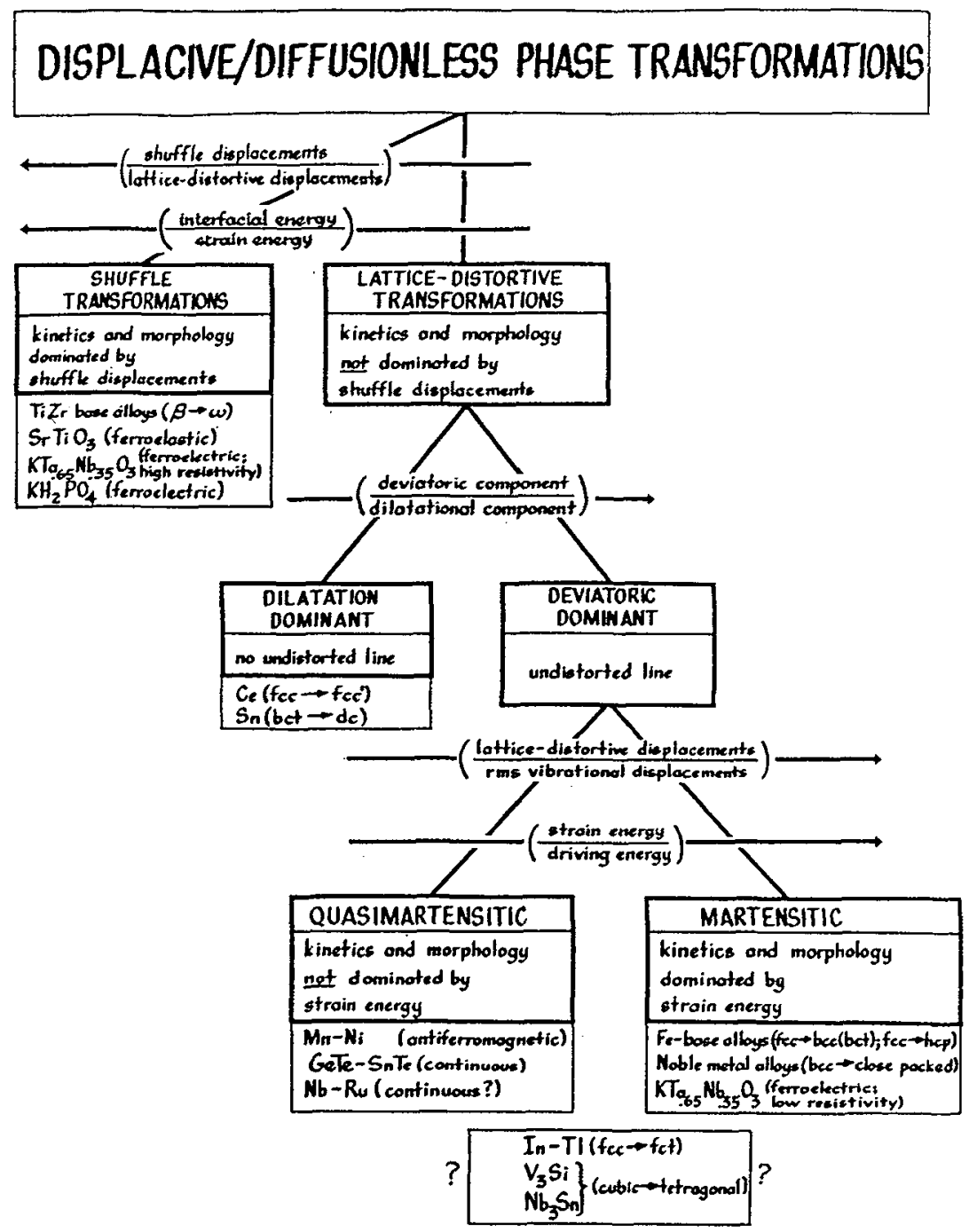

Fig. 2 - The classification of displacive transformations proposed by Cohen, Olson \& Clapp 14 
Khachaturyan 15 - "Martensitic transformations proceed by diffusionless formation of martensitic phase "islands" within a parent phase crystal lattice. Since crystal lattices of the two phases involved differ from each other, for them to fit together requires significant atomic displacements producing elastic strain. The elastic strain may be reduced by a diffusion migration of atoms as in the usual recrystallization processes or by further transformation until location, shape, size, and orientation of the newly formed portions of the martensitic phase are such that a partial relaxation of the elastic strain produced by previously formed martensitic crystals is provided." and also, "Elastic strain and coherent conjugation of the crystal lattices of parent and martensitic phases are the key features of martensitic kinetics and morphology."

Porter and Easterling 16 - "Martensite is a term used in physical metallurgy to describe any diffusionless transformation product, i.e. any transformation in which from start to completion of the transformation individual atomic movements are less than one interatomic spacing. The regimented manner in which atoms change position in this transformation has led to it being termed military, in contrast to diffusion-controlled transformations which are termed civilian. In principle, all metals and alloys can be made to undergo diffusionless transformations provided the cooling rate or heating rate is rapid enough to prevent transformation by an alternative mechanism involving the diffusional movement of atoms. Martensitic transformations can thus occur in many types of metallic and non-metallic crystals, minerals, and compounds."

\section{Conclusion}

It may be noted that all of these definitions at least seem to agree on the matter of a diffusionless transformation (although note C. S. Smith's recent remark ${ }^{17}$ !), even though ironically, very little is yet known about the actual cooperative movements that the atoms are supposed to follow at a moving interphase interface, with the possible exception of the hcp-fcc transformation in Co alloys. However, accepting this article of faith as a basic common ground, one can conclude that martensitic transformations are a subset in the general family of diffusionless transformations, i. e. displacive transformations, (although Porter and Easterling's definition would seem to make the two synonymous!) . Nevertheless, this agreement in theoretical concept does not easily carry over into the laboratory because it is very difficult to prove in many cases that the transformation process was indeed diffusionless. Indirect, somewhat handwaving arguments are generally used in the literature to deal with this question, e. g. the very low temperature at which the transformation occurred, or the speed at which it happened, etc. We shall return to this operational difficulty later, because we have theoretical difficulties to discuss first.

If, for the moment, we accept what seems to be the basic geometrical concept of a martensitic transformation, i. e. a cooperative (diffusionless) motion of atoms across an interface causing a shape change, (and this would appear to be a common point of agreement of all the definitions cited), then there remain at least two major forks in the road of theoretical classification.

The first fork is deciding whether all of the atoms must move in a diffusionless matter, or just some of them? This issue was discussed at some length by Cohen et al. ${ }^{18}$ and is also the question raised by Lieberman's definitions of orthomartensite vs. paramartensite, etc.

One pragmatic way of approaching this decision is: What would be lost by including as martensitic transformations, cases where some of the atomic species are sufficiently fast diffusers to achieve at least short range migration? Would the continuum crystallographic theories become any less applicable? Of course, if we as a scientific community were to agree that the short range diffusion of some atomic species (but not all), still made it possible to refer to a transformation as "martensitic",-then it could be suspected that some Bainitic reactions might be found to qualify. This might be historically embarassing, but perhaps ultimately have a pragmatic unifying benefit.

Second, must the transformation be first order in the Ehrenfest sense, i. e. have parent and product in coexistence, have a latent heat and hysteresis, etc? This question was also agonised over by (among others) Cohen et al., who left the nearly higher order (nearly continuous) transformations (e. g. InTl, $\mathrm{V}_{3} \mathrm{Si}, \mathrm{Nb}_{3} \mathrm{Sn}$, etc.) in a box at the bottom with a question mark (see Fig. 2). This is an awkward point because as the composition of an alloy changes, the transformation may steadily decrease in first order character until it becomes, in fact, continuous in nature. At what stage are we to cease referring to it as martensitic? Most other transformations that 
we classify (e. g. order-disorder, ferromagnetic, gas-liquid-solid, etc.) we do not call by another name when they cease to be first order.

Of course, in the case of martensitic transformations the strain produced between parent and product has from the very earliest studies been considered as perhaps its most practical manifestation through its strengthening effect. However as a system decreases in first order character (as measured by the temperature span of the hysteresis relative to the absolute temperature of transformation, for instance) there is less and less of a coexistence of parent with product, until at the limit of a continuous transformation it is only different variants of the product phase which set up opposing strain fields amongst each other and cause the highly geometric planar "magnetic domain boundary" morphologies. Again, the question may be asked what would we lose by including them under the term "martensitic"? Would the continuum crystallographic theories no longer be applicable? At what point would they fail?

Now, having raised these theoretical debating points, let us return to the experimental realm and readdress the question of how one can even prove if a given transformation involves a cooperative (diffusionless) displacement of atoms from parent to product phase. Various tests are often offered, but none seem to be uniquely satisfying. A partial list would include:

- Surface upheaval on a prepolished surface - but Bainitic and massive reactions often show this feature as well, not to mention the formation of the long-period ordered $\mathrm{CuAu} \Pi$ from the disorderd phase.

- Surface scratches are not interrupted but are continuously deformed when intersected by a plate of the product phase - but Bainitic and massive reactions often show this feature too.

- Low temperature of the transformation - relative to what ?; how low need it be to qualify ?; and more importantly, are there no "high" temperature martensitic transformations possible?

- High speed of the transformation - relative to what? Are "slow" martensitic transformations impossible? Why?

It may be argued that the best test of the presence of a cooperative (diffusionless) displacement of atoms is whether or not coherent acoustic energy is produced. This refers to the emission of ultrasonic energy in some range of frequencies that would be determined by the speed of the transformation front, and is a necessary concomitant of any coordinated movement of atoms over a significant area. Furthermore, it is a relatively simple, inexpensive test which can be applied over a very wide range of temperatures by introducing a thermally insulating quartz rod as an acoustic wave guide between sample and detector. However, care must be taken to search for acoustic emission in the right frequency range, which can be roughly estimated as the velocity of the interface divided by the interatomic spacing.

Of course, acoustic emission also appears in mechanical twinning, large array dislocation motion and fracture propagation, but those cases are clearly distinguishable since no new phase results. It is only that they share the characteristic with a martensitic transformation of involving a cooperative movement of many atoms simultaneously. Other types of phase transformations that occasionally may also produce acoustic energy (e. g. ferromagnetic, ferroelectric) do so through a coupling of their primary order parameter to their lattice displacive modes, but also are not likely to be confused with a martensitic transformation. It does, however, raise the interesting question of whether acoustic emission might not be detected during the formation of lower Bainite, or during massive transformations, or in association with some of the other cases (currently not regarded as martensitic) where surface upheaval is observed.

It is perhaps surprising that more emphasis has not been given in the field to this important characteristic of cooperative atomic displacements, since it has been known for some time that the measurement of acoustic emission is the most sensitive method of detecting the very first martensite events ${ }^{19}$, and a body of excellent work has been done in matching the progress of martensitic transformations in various alloys to the cumulated acoustic emission events ${ }^{20,21}$ (see Figs. 3 \& 4). In fact the process is very analogous to seismic phenomena, and it would seem that much use could be made of the rather advanced analysis methods which geologists employ to determine details of earthquakes by measurements of seismic wave components. This author is aware of only one crude and rather limited "seismic analysis" of a martensitic transformation ${ }^{22}$, but it would seem to promise much for revealing important details about the growth mechanism and the nature of atomic motions at the interface that have, so far, eluded us and yet seem so important for our fully being able to understand the mechanism and kinetics of martensitic transformations. 


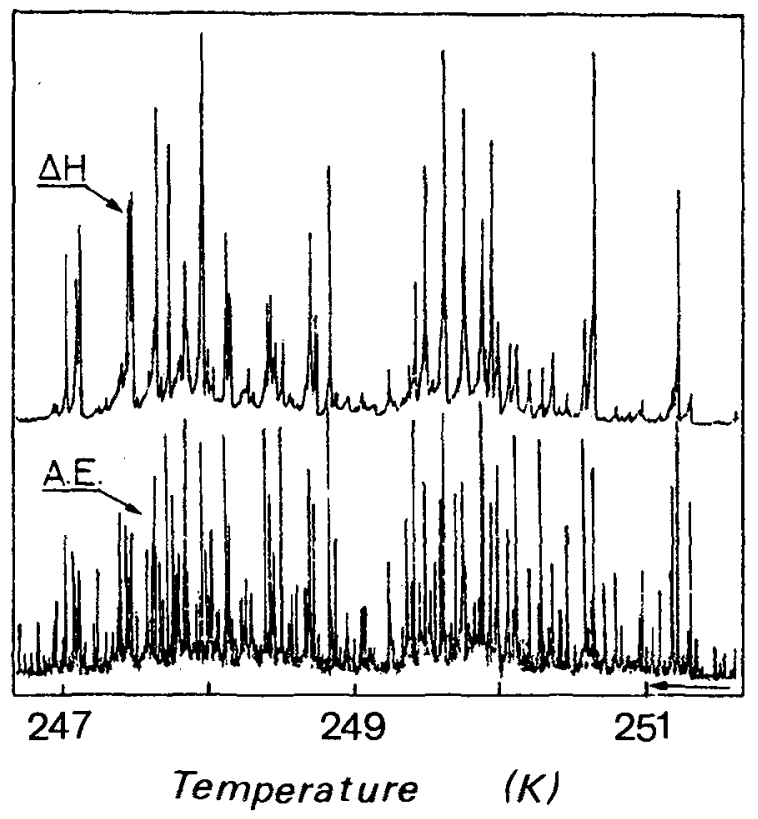

Fig. 3 - Fig. 2 from Planes et al. 20 showing simultaneous acoustic emission and thermal peaks during cooling of a Cu-Zn-Al alloy.

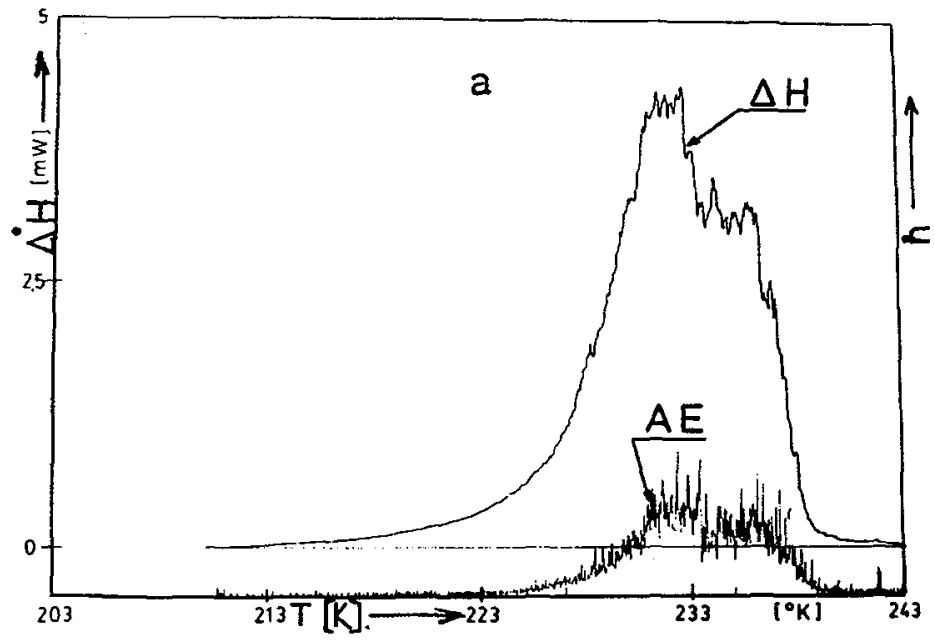

Fig. 4 - Fig. 2a from Rapacioli, Guénin and Macqueron 21 showing acoustic emission and latent heat evolution over an entire transformation cycle in a $\mathrm{Cu}-16 \mathrm{Zn}-16 \mathrm{Al}$ alloy. 
Taking all of these elements into account, if we now return to the central essence of agreement distilled above, $i$. e. that a martensitic transformation involves a cooperative (diffusionless) motion of atoms across an interface causing a shape change and if we can further agree that for a martensitic transformation:

1) Not all species (or subsets) of atoms need to move in a diffusionless way, and

2) There must be an interface between parent and product then, by the addition of two short qualifiers, one has as a suggested definition:

A martensitic transformation involves a cooperative motion of $a$ set of atoms across an interface causing a shape change and sound.

Apart from being compact, this definition offers the three tests: $i$ ) observation of a parent/product interface, $i$ ) appearance of shape change, and iii) detection of acoustic emission, to directly resolve the questions of whether the transformation is sufficiently first order and latticedistortive to be called martensitic, and whether cooperative (diffusionless) motion has, in fact, occurred for at least some atomic species. It is assumed that all the other usual concomitants of a martensitic transformation (surface upheaval, invariant plane strain, heterogeneous character, existence of a habit plane and an orientation relationship, latent heat, hysteresis, etc.) will naturally follow if the basic conditions of the definition outlined above are satisfied. In short, it is hoped that this definition includes a minimum set of elements necessary to define a martensitic transformation, but no more than are truly needed.

Finally, the answer to the somewhat facetious question raised in the title of this paper perhaps may be: we will know it by the noise it makes!

\section{Acknowledgements}

It is a pleasure to note many wonderful (and noisy) discussions over the years with, among others: Morris Cohen and John Cahn who got me intrigued with "martensitic transformations" in the first place; Greg Olson and Jack Christian who always had an amazing grasp of the crystallographic complexities; Gerard Guénin, Rolf Gotthardt, Lee Tanner and Steve Shapiro who knew so much about the experimental aspects of martensitic transformations; Jim Krumhansl and Si Moss who brought a physicist's sense of wonder to the subject; and to Jon Rifkin and all of my graduate students and other co-workers for their insights, inspiration (and perspiration) in persevering together through some long, dark (but interesting) nights. The research support of NSF, DOE and the UConn Research Foundation has been very helpful and much appreciated.

\section{References}

${ }^{1}$ Seminal observations were reported by H. C. Sorby (1864), D. K. Tschernoff (1866), A. Martens (1878); see the excellent review article "Early Ideas on the Structure of Steel" by C. S. Smith in "Martensite", G. B. Olson and W. S. Owen, eds., ASM International, Metals Park, Ohio (1992) pp. 21-39; although Martensite was named after A. Martens by M. F. Osmond in 1895 in the manner of naming a mineral after its discoverer, probably the honor should instead have been conferred on Sorby e.g. "Sorbyite".

${ }^{2}$ M. F. Osmond, Arts Chimiques 94, 480 (1895)

$3_{G}$. V. Kurdjumov, Phys. Z. der Sowietunion, 4, 488 (1933)

${ }^{4}$ M. S. Wechsler, D. S. Lieberman and T. A. Read, Trans. AIME 197, 1503 (1953)

5J. S. Bowles and J. K. Mackenzie, Acta Metall. 2, 129, 138, 224 (1954)

6 A. G. Khachaturyan, Sov. Phys. Solid State 8, 2163 (1967)

7J. M. Ball and R. D. James, Arch. Rat. Mech. Anal. 100, 13 (1987)

8B. A. Bilby and J. W. Christian, in "The Mechanism of Phase Transformations in Metals", Institute of Metals, London, (1956) see p. 123

${ }^{9}$ L. Kaufman and M. Cohen, Progress in Metal Physics 7, 165-246 (1958) see p. 171

${ }_{10}$ J. W. Christian, "The Theory of Transformations in Metals \& Alloys" 1 st Ed'n, Pergamon, Oxford (1965) see p. 803 
${ }^{11}$ D. S. Lieberman in "The Mechanism of Phase Transformations in Crystalline Solids", The Institute of Metals, London (1969) p.168

${ }^{12}$ H. M. Clark and C. M. Wayman, in "Phase Transformations", H. I. Aaronson, ed., ASM, Metals Park, Ohio (1970), see p. 110

13Z. Nishiyama, in "Martensitic Transformation", Academic Press, NY (1978), see p. 11

${ }^{14}$ M. Cohen, G. B. Olson and P. C. Clapp, Proc. ICOMAT-79, M. I. T., Cambridge, MA (1979) see p. 10

15 A. G. Khachaturyan,"Theory of Structural Transformations in Solids", J. Wiley \& Sons, NY (1983), see p. 157-8

${ }^{16}$ D. A. Porter and K. E. Easterling, "Phase Transformations in Metals and Alloys", Chapman and Hall, London (1981) see p. 382

17 op. cit. C. S. Smith in "Martensite", G. B. Olson and W. S. Owen, eds., ASM International, Metals Park, Ohio (1992) pp. 21-39, who says on p. 36-37: "The formation of martensite is not diffusionless; it involves the directed movement of space in a way somewhat analogous to the movement of neutrinos in nuclear transformations."

${ }^{18}$ op. cit. M. Cohen, G. B. Olson and P. C. Clapp, Proc. ICOMAT-79, M. I. T., Cambridge, MA (1979) pp. 1-11

19 see for example, S. S. Hsu, J. H. Chen and P. C. Clapp, phys. stat. sol. (a). 50:393 (1978)

20 A. Planes, J. L. Macqueron, M. Morin, G. Guénin and L. Delaey, J. de Physique 43, C4-615 (1982)

${ }^{21}$ R. Rapacioli, G. Guénin and J. L. Macqueron, Proc. ICOMAT-86, pp. 204-209

22Z-Z Yu, and P. C. Clapp, Met. Trans. A, 20A, 1601-1615 (1989) 